\title{
BMJ Open Feasibility randomised controlled trial of remote symptom chemotherapy toxicity monitoring using the Canadian adapted Advanced Symptom Management System (ASyMS-Can): a study protocol
}

Saeed Moradian (10 , ${ }^{1}$ Monika Krzyzanowska, ${ }^{2,3}$ Roma Maguire, ${ }^{4}$ Vishal Kukreti, ${ }^{5}$ Eitan Amir, ${ }^{6}$ Plinio P Morita, ${ }^{7}$ Geoffrey Liu, ${ }^{8,9}$ Doris Howell ${ }^{6}$

To cite: Moradian S, Krzyzanowska M, Maguire R, et al. Feasibility randomised controlled trial of remote symptom chemotherapy toxicity monitoring using the Canadian adapted Advanced Symptom Management System (ASyMS-Can): a study protocol. BMJ Open 2020;10:e035648. doi:10.1136/ bmjopen-2019-035648

- Prepublication history for this paper is available online. To view these files, please visit the journal online (http://dx.doi. org/10.1136/bmjopen-2019035648).

Received 14 November 2019 Revised 15 April 2020 Accepted 29 April 2020
Check for updates

(C) Author(s) (or their employer(s)) 2020. Re-use permitted under CC BY-NC. No commercial re-use. See rights and permissions. Published by BMJ.

For numbered affiliations see end of article.

Correspondence to

Dr Doris Howell;

doris.howell@uhn.ca

\section{ABSTRACT}

Introduction Technology is emerging as a solution to develop home-based, proactive 'real-time' symptom monitoring and management in cancer care. The Advanced Symptom Monitoring and Management System-Canada (ASyMS-Can) is a remote phone-based symptom management system that enables real-time remote monitoring of systemic chemotherapy toxicities.

Methods and analysis This study is an open-label, prospective, mixed-method, Phase II, 2-arm parallel group assignment (ASyMS-Can vs usual care) feasibility study in patients with cancer receiving systemic (neoadjuvant or adjuvant) chemotherapy at Princess Margaret Cancer Centre. A total of 114 patients will be recruited in oncology clinics prior to initiation of chemotherapy. Patients in both arms will complete a demographic and a set of questionnaires at enrolment, mid and end of treatment. Patients in intervention arm will be provided with an encrypted, secure, preprogrammed ASyMS phone for symptom reporting daily for the first 14 days of each chemotherapy treatment cycle up to sixth cycle (16 weeks). Feasibility metrics (recruitment, retention and protocol adherence) and outcomes to assess impact of ASyMS - Can include symptom severity, emotional distress, quality of life and acceptability to patients and clinicians.

Ethics and dissemination The study has received ethical and institutional approvals from the University Health Network. Dissemination will include presentations at national/international conferences, and publications in peer-reviewed journals.

Trial registration number NCT03335189.

\section{INTRODUCTION}

Treatment toxicities due to systemic chemotherapy are prevalent ${ }^{1}$ and often underrecognised, ${ }^{2}$ resulting in high rates of symptom distress, avoidable visits to the emergency department (ED) and hospitalisations. ${ }^{34}$ Empirical evidence has substantiated

\section{Strengths and limitations of this study}

- In the present randomised controlled clinical trial, an electronic patient-reported outcomes for management of chemotherapy-related toxicities is implemented.

- Both qualitative and quantitative research methods were applied to identify any procedural and clinical uncertainties including estimates of recruitment and retention rates, and feasibility and acceptability of the intervention.

- Findings may not be generalisable due to a singlecentre clinical trial and lack of an attention control group.

the role of remote symptom monitoring in ambulatory cancer care, using electronic patient-reported outcomes (ePROs), for symptom management. ${ }^{5} 6$ Several ePRO systems exist, ${ }^{6-10}$ and have been demonstrated to improve patient satisfaction, patient-clinician communication, health-related quality of life (HRQoL), overall survival, and reduce acute health service utilisation. ${ }^{511-13}$ These systems facilitate using evidence-based guidelines as part of a comprehensive symptom management approach in cancer care. ${ }^{14} \mathrm{~A}$ recent study also showed the cost-effectiveness of an ePRO symptom monitoring programme for patients with advanced solid tumour in Alberta-Canada and recommended implementation of ePRO tools to support management of chemotherapy-related toxicities in Canadian patients with cancer. ${ }^{15}$ However, few systems use specific features such as decision support for standardising symptom triage $^{10}$ or provide automated self-care information that may improve the level of patient 
activation. ${ }^{916}$ Growing evidence suggests that patient activation plays an imperative role for enabling better coping with and managing cancer-related symptoms. ${ }^{17} 18$

The ASyMS is a mobile phone based system that enables real-time remote monitoring of systemic chemotherapy toxicities, using an ePRO measure aligned with Common Terminology Criteria for Adverse Events. ${ }^{19}$ ASyMS was developed and tested in Europe ${ }^{20}$; however, based on best practices for writing actionable statements for selfcare advice to support patient activation in symptom selfmanagement ${ }^{7}$ and ASyMS usability study data in Canadian patients with cancer, ${ }^{18}$ we enhanced the self-care advice. Additionally, we adapted the risk scoring system and decision-support algorithms within the device to align with Canadian evidence-based protocols for symptom triage $^{21}$ and for consistency with definitions for fever in provincial guidelines. $^{22}$

ASyMS-Can supports daily remote symptom reporting and back-end analytics of ePROs to derive risk scores by combining symptom severity with other data that triggers alerts that are sent to clinical trial nurse phones to prompt early intervention in managing cancer patients receiving chemotherapy. ASyMS also provides automated self-care advice to empower patients to take a more active role in self-management; thus has the potential to reduce severity of symptoms. While ASyMS has utility in Europe, evaluation of its feasibility and acceptability in 'real world' Canadian ambulatory oncology practices prior to large scale trials are needed. Feasibility studies provide data about whether a study can be done and estimate key parameters to design a larger, definitive trial. ${ }^{23}$ This study is a mixed-method, prospective, Phase II, 2-arm parallel group assignment (ASyMS-Can vs usual care) feasibility trial in patients receiving systemic neo-adjuvant or adjuvant chemotherapy for early stage (Stages I-III) breast, colorectal, and lymphoma (Hodgkin's), non-Hodgkin's malignancies.

\section{AIMS}

The overall aim of this research project is to improve the management of cancer treatment-related toxicities by early identification of symptoms and prompting intervention using a mobile phone-based technology. The aims of this feasibility study are as follows:

1. To assess recruitment/retention rates and adherence to the intervention. Rates, reasons and factors associated with attrition, in both study arms, will be examined. Recruitment rate aim is 7-8 patients/month, based on other studies. ${ }^{25}$ A composite score for adherence rate (ratio of completed ePRO/ratio of daily ePRO reports that should be completed), number of alerts ( (amber or red) per cycle) will be calculated.

2. To evaluate acceptability of ASyMS-Can and explore the views and experiences of the intervention in a sample of cancer patients and clinicians.

3. To estimate whether, compared with the control group, the ASyMS-Can intervention impacts on outcomes:
- Reduced symptom severity measured by Memorial Symptom Assessment Scale (MSAS). The MSAS is a valid and reliable instrument ${ }^{26}$ and includes 24 items for report on whether a symptom (eg, pain, lack of energy and shortness of breath) occurred during the previous week, as well as any distress it may have caused.

- Reduced psychological distress measured by Depression, Anxiety and Stress Scale (DASS21) at three time points (baseline, midpoint of treatment cycles and end of treatment cycles). DASS21 contains 21 items for self-reporting for measuring a range of symptoms common to both depression and anxiety. It has high internal consistency (Cronbach's alpha scores of $>0.7$ ) and significantly correlates with other measures. ${ }^{27}$

- Improved self-efficacy for coping measured by Cancer Behavior Inventory (CBI-B) at baseline, midpoint of treatment cycle, and end of treatment. CBI-B is a valid and reliable instrument with 12-items designed to assess coping self-efficacy of cancer patients and takes approximately 2 min to complete. ${ }^{28}$

- Improved HRQoL measured by EuroQual-5D-5L (EQ-5D-5L) at three time points (baseline, midpoint of treatment cycles and end of treatment cycles). EQ-5D-5L a simple and relatively quick instrument for patient completion which has been validated in a diverse patient population including patient groups with chronic conditions. ${ }^{29}$

In addition, the following outcomes will be assessed:

- Health service utilisation will be measured by a selfreport questionnaire and include information such as days in hospital, ED visits, urgent care use, unscheduled clinic visits and will be collected at baseline, midpoint and end of treatment. It will be assessed as a binary variable and for comparison in odds between groups at midpoint and end of treatment.

- Participant's satisfaction with using the ASyMS-Can will be measured by The Post-Study System Usability Questionnaire (PSSUQ) at the end of intervention. The PSSUQ is a research instrument that consists of 16 items designed to assess users' perceived satisfaction with computer systems or applications. ${ }^{30}$

\section{METHODS}

\section{Study design}

This study is an open-label, prospective, mixed-method, Phase II feasibility study with parallel randomisation (1:1) of participants into the mobile remote monitoring intervention arm or a usual care arm (RCT (randomised controlled trial)). The protocol is written in compliance with the Standard Protocol Items: Recommendations for Interventional Trials and its checklist. ${ }^{31}{ }^{32}$ On completion of the study, clinicians and patients in the intervention arm are invited to participate in interviews/focus groups based on a qualitative descriptive study design ${ }^{33}$ to gain a more in-depth understanding of acceptability of the intervention, outcomes measures, and implementation 


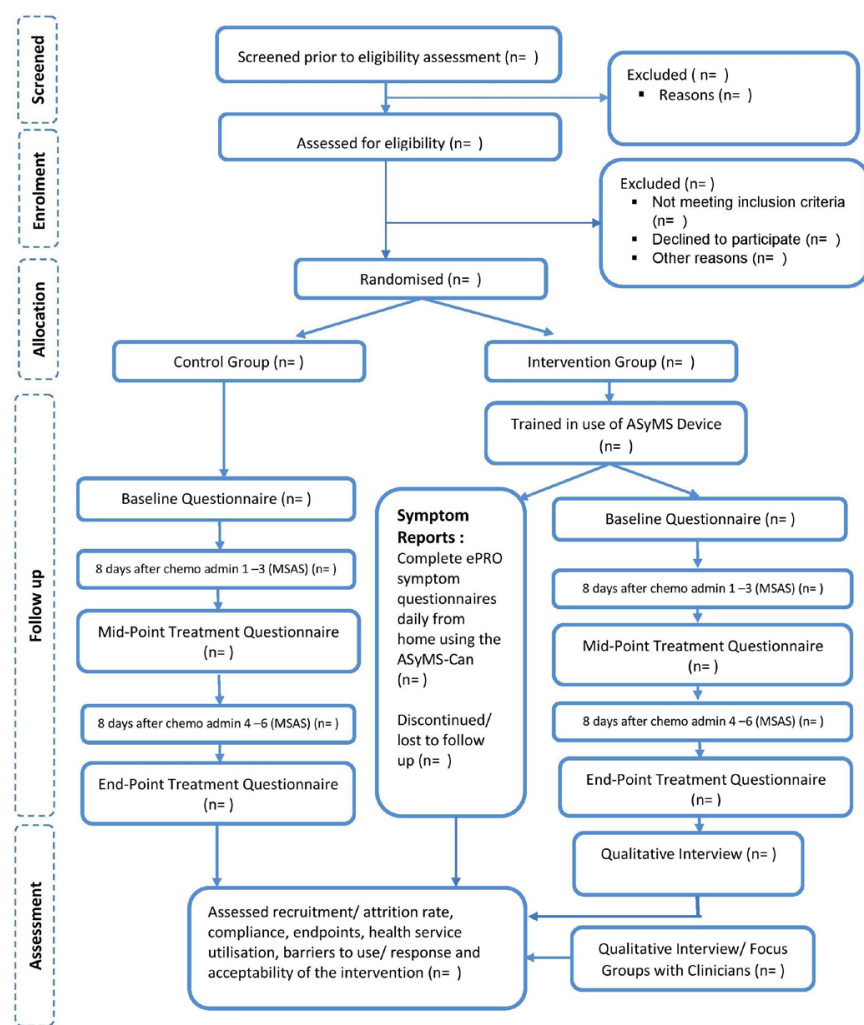

Figure 1 Flow diagram of study design. ASyMS, Advanced Symptom Monitoring and Management System; ePRo, electronic patient-reported outcome; MSAS, Memorial Symptom Assessment Scale.

barriers to use of the ASyMS-Can mobile device. Figure 1 outlines the flow of the study.

\section{Setting}

The study is undertaken at the Princess Margaret Cancer Centre (PM), which is an academic research centre and teaching hospital, affiliated with the University of Toronto Faculty of Medicine as part of the University Health Network (UHN), Toronto, ON, Canada. The Institutional Review Board Approval was obtained from the Research Ethics Board (REB) of UHN to conduct the study.

\section{Participant}

\section{Inclusion}

1. Adults patients ( $\geq 18$ years old) diagnosed with early stage breast, colorectal and lymphoma.

2. Scheduled to receive a minimum of two cycles of systemic chemotherapy in 2-weekly, 3-weekly or 4-weekly cycles (ie, administered at repeated cycles of 14, 21 or 28 days, respectively).

3. Ability to use or be trained in use of a mobile phone for symptom reporting and able to complete questionnaires in English.

\section{Exclusion}

1. Enrolled/receiving an investigational treatment.

2. Scheduled to receive concurrent radiotherapy or a weekly chemotherapy protocol.
3. Any distant metastasis or receiving ONLY hormonal therapy, oral chemotherapy, targeted agents and monoclonal antibody/PD-1 (programmed cell death)/PDL1 (programmed cell death receptor ligand) inhibitors.

4. Cognitive impairment assessed by the treatment team which may impede completion of study measures and unable to perform self-care (ECOG (Eastern Cooperative Oncology Group) $\geq 3$ ) .

5. Unable to provide written informed consent.

\section{Sample size}

A one-sided type I error rate of 0.10 and type II error rate of 0.15 (power of $85 \%$ ) are reasonable statistical parameters for a feasibility study. ${ }^{34}$ A sample size of 88 subjects (44 per group) is required to test the difference of $0.5 \mathrm{SD}$ change $^{35}$ at midpoint of cycles using a two-sample t-test. Accounting for a $30 \%$ drop out rate, ${ }^{25}$ a total sample of 114 patients will need to be recruited. This sample size will allow us to estimate a recruitment rate of $70 \%$ to within a $95 \%$ CI of $\pm 10 \%$ and to calculate an effect size for the main trial. ${ }^{36}$ Feasibility study sample sizes range from 24 to 50 for estimation of variance in outcomes with precision in feasibility studies (low $\mathrm{SE}>0.1$ ). ${ }^{37} 38$

\section{Patient recruitment/randomisation}

Patients are screened through the Pathways Healthcare Scheduling, Electronic Patient Record (EPR), as well as outpatient lists. Eligible patients are recruited prior to initiating treatment in medical oncology consultation appointments. All patients will be informed that they will be randomly allocated to either the 'mobile phone arm' or 'usual care arm.' Consented participants are randomised in equal numbers to one of two groups with stratification by cancer type to ensure balance in groups. Randomisation will be done centrally by the PM Biostatistics department using a SAS computerised randomisation process in permutated blocks (random blocks of varying size). Those who decline to participate will be asked for verbal consent to collect basic information (eg, age, education and type of cancer) and reasons for refusal. Patients who consent to study participation will be given the baseline questionnaire to complete prior to randomisation. Research staff who collected the data and clinicians are not blinded to group assignment given the nature of the intervention.

\section{Intervention}

Participants allocated to the intervention group will be provided with the encrypted, secure, pre-programmed ASyMS-Can phone and instructed of its use; how to report their symptomatology on a daily basis using the ASyMS symptom questionnaire (Chemotherapy Toxicity SelfAssessment Questionnaire (CTAQ). ${ }^{19}$ The CTAQ assesses ten chemotherapy-related symptoms with an additional option to report up to six further symptoms. Data reported by the participants will be sent to a secure, encrypted PM clinical central server hosting the risk-alerting algorithms and clinical symptom platform. When patients send their 


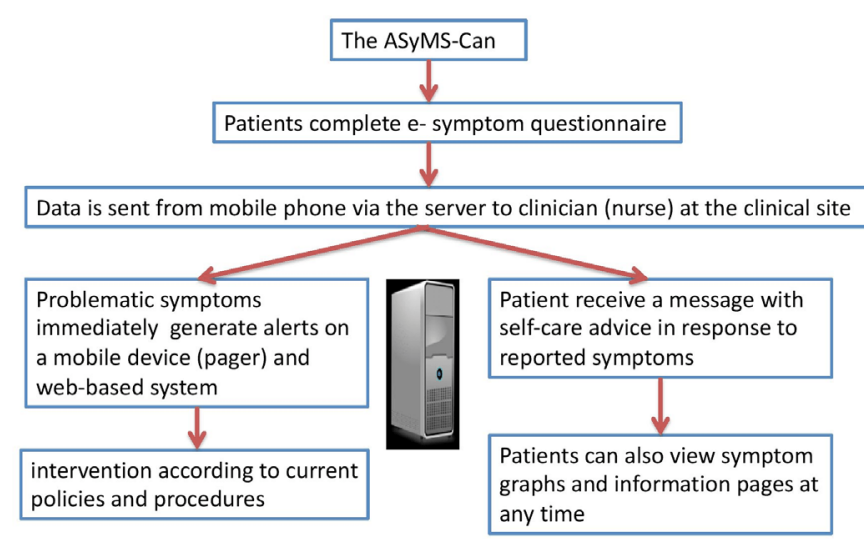

Figure 2 ASyMS-Can monitoring system. ASyMS-Can, Advanced Symptom Monitoring and Management SystemCanada.

symptoms data, they immediately receive evidence-based self-care advice for the specific symptoms reported.

In addition, patients can access a self-care library and symptom graphs (detailing trends in individual symptoms experienced) through the ASyMS-Can patient phones. If the incoming symptom reports are of clinical concern, the server software will generate two levels of alerts (amber and red) that will be sent to the designated nurse, who will receive alerts on a dedicated ASyMS-Can nurse handset (mobile phone). The nurse will view the patient's symptom reports on a secure web page, and contact the patient directly at home by telephone, guided through a decision-support algorithm on the web-based platform to systematise the triage based on the COSTARS guideline, ${ }^{39}$ facilitating the initiation of 'real-time' clinical interventions. An 'amber alert', which requires response within 4 hours, indicates that the symptom(s) are not severe or life-threatening but early intervention might prevent further symptom progression. The second level of the triage alert, 'red alert', will be sent to the nurse for severe symptoms and will require response within $30 \mathrm{~min}$ of receipt of the alert (figure 2).

\section{Data collection}

Socio-demographic and clinical/disease characteristics will be collected at baseline. Participants from both arms will complete self-report measures to assess the participants' physical and psychological symptoms, quality of life and healthcare utilisation (table 1). Patient outcomes will be measured at baseline, 2 weeks after completion of cycle 3 , and 2 weeks after completion of last cycle (up to a maximum of six cycles) to determine suitability and timing of the primary endpoint for the main trial. All participants will also be contacted over the phone to complete the MSAS about 8 days after each treatment cycle. This timeframe was selected based on the typical pattern of symptom distress from our previous PRO study $^{38}$ and other studies that have estimated appropriate time-points for capturing 'true symptom burden' during treatment in mixed cancers. Data collection measurements and time points are shown in table 1.
Patients in the intervention group will be asked to use the ASyMS-Can mobile phone to report their symptoms for the first 14 days of each treatment cycle until end of the final cycle of treatment.

\section{Training/education}

Both control and intervention participants will receive the standard preparatory training (usual treatment) of how to manage their chemotherapy symptoms by their clinic nurses and/or pharmacists. All participants will receive patient education materials and the standard toxicity monitoring care at PM clinics.

\section{Intervention arm}

Patients in the intervention arm will receive a training session to learn how to use the ASyMS-Can mobile phone to report their symptoms. Patients will be instructed to complete symptom reports at least once a day in the morning before 12:00 and whenever they feel unwell. Further training will be provided if a patient experiences any problems using the phone. The training will be provided by the research coordinator. In addition, a manual with instructions on how to use the phone will be given to each patient. Patients will also be instructed that nurses will not receive alerts during the evening hours or night (after 17:00), on weekends/holidays, or in case of system failure. They will be instructed in these cases to follow clinic team advice as standard care will apply.

\section{Control arm}

Patients in control arm will be informed about data collection procedures and how to complete the questionnaires at different time points.

\section{Nurses training}

Clinical trials nurses, designated for trial management in each cancer site, will receive training on computers as to how to use the device and platform with additional oneto-one training by the research coordinator to respond to the patients alerts during business hours.

A standard operating procedure for clinical trials nurses to respond, handle alerts, communicate, and collaborate with the clinical and research team is developed. On receipt of an alert, the nurse reviews the symptom report, clinical and demographic information, as the first step in response on the ASyMS-Can web-based system. In addition, trial nurses can also review the patients EPR and then phones the patient to further assess, using evidence-based telephone triage protocols ${ }^{39}$ and determines the appropriate disposition (urgent vs non-urgent) and directs the patient accordingly. In the case of amber alerts, the nurse will make their own assessment using information provided in ASySM-Can and can elect to close the alert using their professional judgement and without running through the initial alert handling protocols. However, in the case of any red alerts the nurse will handle the alert and inform the most responsible physician to ensure all appropriate actions are taken. All actions and interventions will be documented in the ASyMS-Can system and 
Table 1 Schedule of data collection measurement

\begin{tabular}{|c|c|c|c|c|c|c|}
\hline $\begin{array}{l}\text { Assessment/ } \\
\text { measurement } \\
\text { instrument }\end{array}$ & $\begin{array}{l}\text { Prior to/on } \\
\text { enrolment }\end{array}$ & Baseline & $\begin{array}{l}\text { Mid cycle } \\
\text { ( } 8 \text { days after } \\
\text { administration } \\
\text { of each chemo } \\
\text { cycle) } \\
\text { over the phone }\end{array}$ & $\begin{array}{l}\text { Mid treatment } \\
\text { ( } 2 \text { weeks } \\
\text { after third } \\
\text { administration of } \\
\text { chemo cycle) }\end{array}$ & $\begin{array}{l}\text { End treatment } \\
\text { ( } 2 \text { weeks } \\
\text { after last } \\
\text { administration of } \\
\text { chemo cycle) }\end{array}$ & $\begin{array}{l}\text { After } \\
\text { completion of } \\
\text { the study } \\
\text { (within } \\
\text { 7-10 days) }\end{array}$ \\
\hline Eligibility checklist & $\mathbf{x}$ & & & & & \\
\hline $\begin{array}{l}\text { Demographic } \\
\text { variables }\end{array}$ & $\mathbf{x}$ & & & & & \\
\hline $\begin{array}{l}\text { Clinical } \\
\text { characteristics }\end{array}$ & $x$ & & & & & \\
\hline $\begin{array}{l}\text { Depression } \\
\text { Anxiety Stress } \\
\text { Scale (DASS21) }\end{array}$ & & $x$ & & $x$ & $x$ & \\
\hline $\begin{array}{l}\text { Self-Efficacy for } \\
\text { Coping (CBI-B) }\end{array}$ & & $\mathbf{x}$ & & $x$ & $x$ & \\
\hline $\begin{array}{l}\text { HRQoL (EQ-5D- } \\
5 \mathrm{~L})\end{array}$ & & $x$ & & $x$ & $x$ & \\
\hline $\begin{array}{l}\text { Health Care } \\
\text { Utilisation } \\
\text { (self-report } \\
\text { questionnaire) }\end{array}$ & & $x$ & & & $x$ & \\
\hline $\begin{array}{l}\text { Patients' } \\
\text { experience- } \\
\text { interview }\end{array}$ & & & & & & $\mathbf{x}$ \\
\hline $\begin{array}{l}\text { Clinicians' } \\
\text { experience-focus } \\
\text { group }\end{array}$ & & & & & & $\mathrm{x}$ \\
\hline
\end{tabular}

ASyMS, Advanced Symptom Monitoring and Management System; CBI-B, Cancer Behavior Inventory; EQ-5D-5L, EuroQual-5D-5L; HRQoL, health-related quality of life; PSSUQ, Post-Study System Usability Questionnaire.

Clinical Research Record in the patient's chart. Therefore, all clinicians have access to these documentations through EPR.

\section{Qualitative interviews and focus groups}

At study completion (within 7-10 days), all participants in the intervention group will be invited to participate in an audio-taped semi-structured interview. We aim to have a total of 12-16 patient participants, as it is proposed that saturation most often occurs around 11-15 participants in homogeneous groups. ${ }^{40}$ Also, all clinicians exposed to patients who used the mobile device will be invited to provide feedback to increase our understanding of the feasibility and care processes; implementation barriers in work flow impeding timely response to alerts; evaluate acceptability for use in routine practice and explore contexts and mechanisms in action, as well as implications of the ASyMS-Can in clinical practice. The leading sites members in PM who are involved with the ASyMS-Can intervention will be contacted to make initial contact to potential clinician participants. The leading sites members will inform their staff about the study and give them the research team's contact information to 
contact them if they are interested to participate in focus group sessions. Those who are interested will then receive the appropriate consent form and will be provided with as much as time needed to consider participation prior to participation in the focus group. In each focus group interview, the researcher will use an interview guide to facilitate discussion among the participants and allow them to freely share their common thoughts/experiences/concerns ${ }^{42}$ Clinicians' focus groups (5-8 healthcare professionals per group) will last approximately $45 \mathrm{~min}$, while the patients' one-on-one audio-taped semistructured interviews will last about $30-45 \mathrm{~min}$.

\section{Treatment duration}

Participants remain on the study until end of chemotherapy treatment cycles (up to six cycles) or discontinuation of cancer treatment, voluntary withdrawal or death.

\section{Patient and public involvement}

We work with patient advocacy groups at UHN. A patient with cancer from this group was involved to read and complete the study questionnaires. The valuable input we received from the patient helped in selecting appropriate measurement tools for this study in terms of using plain language and the time needed to complete them. We aim to elicit participant experiences, thoughts, feelings and satisfaction with the ASyMS-Can by interviewing them after completion of the intervention. Patients are not involved in the recruitment to this study.

\section{Data analysis plan}

Data will be coded and entered separately into an SPSS-12 database. Descriptive statistics will be used to examine baseline equivalence between groups and for calculating adherence, recruitment, retention rates and assessing differential attrition. Univariate and multivariate regression methods appropriate to the data will be used to assess relationships between independent variables and dependent outcome variables. We will examine between and within group effects using generalised linear mixed models on MSAS symptom severity over time controlling for baseline severity scores and covariates (age and sex) to examine the plausible estimates of the effect based on confidence intervals to inform decision-making for proceeding to the main trial. A repeated measure analysis and a midpoint comparison using t-tests or ANOVA (analysis of variance) will be conducted for other outcomes (QoL, self-efficacy and distress) based on data type.

T-tests will be used to compare QoL outcomes symptom severity between arms at the midpoint and

All taped interviews and focus groups will be transcribed verbatim and with data from observations entered into a qualitative data management software programme (NVivo V.8) to facilitate coding, sorting and refining of subcategories and themes. Qualitative interview data will be an inductive thematic content analysis approach based on Graneheim and Lundman. ${ }^{43}$

\section{Ethics and dissemination}

All related study documents reviewed by UHN REB and ethical approval was obtained. The study is explained to all potential participants and clarifies that they are under no obligation to participate, and there will be no negative consequences if they do not agree. If they agree to participate, they are then told they may decline any question and/or withdraw at any stage of the study. Traditional dissemination methods (ie, publications in peer-reviewed journals and conference presentations) will be used to disseminate findings of this study.

\section{Trial management and risks to safety}

The intervention does not replace normal clinic contact but enhances usual care. Any changes to the conduct of the study or to the protocol will be amended and approved by the REB before implementation, unless required to eliminate an immediate hazard to participants.

Should an error occur in the transmission of patient side-effect data, from the patient phone to the system server/website, the software application will advise the patient of the failure of data transmission, and based on alerting algorithms, provide detailed instructions on who and when to call. The server containing the system will be monitored daily for performance and any faults addressed by technicians. Back-up systems are in place for power failures and patients will be advised of system failures, and who and when to call.

A Trial Management Committee (TMC) will be responsible for trial oversight including regular assessment of study conduct, recording and monitoring of risks to safety and adverse events, review accumulating study data related to the safety and efficacy of the study intervention and ensure continued scientific validity and merit of the study. This will provide a level of protection for the participants and the integrity of the trial. If concerns are raised about the conduct or participation of an independent member, then these concerns will be discussed in the TMC meetings.

\section{DISCUSSION}

The technology in ASyMS was developed based on systematic reviews of cancer symptom problems and through extensive engagement with clinicians and patients and has been shown to support symptom management in the UK health system. ${ }^{20} 44$ However, its effects on patient outcomes is uncertain and is being tested in a large multisite trial in European countries. ${ }^{45}$

Based on our systematic review of cancer selfmanagement ${ }^{8}$ and ASyMS usability study data from patients and clinicians in Ontario, ${ }^{46}$ we have modified some of the content in ASyMS for customisation to the Canadian cancer system and the intervention approach. The intervention emphasises the use of a mobile device and its actionable self-care advice to build patients knowledge, skills and confidence in managing health and the effects of cancer treatment and a systematic, structured 
clinician response to alerts using the Canadian evidencebased toxicity triage decision support tools, which have been tested in chemotherapy clinics throughout Canada. ${ }^{47}$

The study will determine the feasibility of recruitment, retention, compliance and implementation barriers in response to alerts and acceptability for use in 'real-world' ambulatory oncology practices in Ontario. Also, the findings of this study will provide plausible estimates of effect of the ASyMS-Can intervention on reducing symptom burden, health services utilisation, and improving patient self-efficacy and acceptability thereby providing the necessary information to design methods and procedures and to inform decisions on proceeding to the full Phase III trial if the results of the study are favourable. Despite a lack of consensus on estimating preliminary effects of an intervention on outcomes in feasibility and pilot studies due to the small samples and possible imprecision, a preliminary estimate of effects alongside clinical considerations is often used to inform decision making regarding the plausible effects that are important for deciding on whether to proceed to the full main trial. ${ }^{48}$ Finally, we will explore the influence of variability in treatment cycles and intensity of treatment regimes in the sample on estimates of effect to inform methods and procedures for the future trial.

\author{
Author affiliations \\ ${ }^{1}$ School of Nursing, York University Faculty of Health, Toronto, Ontario, Canada \\ ${ }^{2}$ University of Toronto Institute of Health Policy Management and Evaluation, \\ Toronto, Ontario, Canada \\ ${ }^{3}$ Department of Medical Oncology and Hematology, Princess Margaret Cancer \\ Centre, University Health Network, Toronto, ON, Canada \\ ${ }^{4}$ University of Strathclyde Department of Computer and Information Sciences, \\ Glasgow, UK \\ ${ }^{5}$ Division of Medical Oncology and Hematology, University of Toronto Faculty of \\ Medicine, Toronto, Ontario, Canada \\ ${ }^{6}$ Department of Medical Oncology and Hematology, Princess Margaret Cancer \\ Centre, University Health Network, Toronto, Ontario, Canada \\ ${ }^{7}$ School of Public Health and Health Systems, University of Waterloo, Waterloo, ON, \\ Canada \\ ${ }^{8}$ University Health Network and Princess Margaret Cancer Centre, Toronto, Ontario, \\ Canada \\ ${ }^{9}$ Epidemiology Division, Dalla Lana School of Public Health, University of Toronto, \\ Toronto, ON, Canada
}

Acknowledgements We would like to thank the Canadian Cancer Society for providing funding for this study. We extend a special thank you and note of appreciation to all patients who participated in this study.

Contributors SM and DH are the main authors of the study protocol. All authors contributed to the design and structure of this research. DH and MK were principal investigators and secured the funding of this study. SM and DH drafted the manuscript. MK, RM, VK, EA, PPM and GL critically revised the manuscript.

Funding This study is funded by the Canadian Cancer Society (CCS) Innovation Grants. Grant number: INNOV17-1/7 05165.

Disclaimer The funder has no involvement in the study design; collection, management, analysis and interpretation of data; writing of the report; and the decision to submit the report for publication.

Competing interests None declared.

Patient and public involvement Patients and/or the public were not involved in the design, or conduct, or reporting or dissemination plans of this research.

Patient consent for publication Not required.
Provenance and peer review Not commissioned; externally peer reviewed.

Open access This is an open access article distributed in accordance with the Creative Commons Attribution Non Commercial (CC BY-NC 4.0) license, which permits others to distribute, remix, adapt, build upon this work non-commercially, and license their derivative works on different terms, provided the original work is properly cited, appropriate credit is given, any changes made indicated, and the use is non-commercial. See: http://creativecommons.org/licenses/by-nc/4.0/.

ORCID iD

Saeed Moradian http://orcid.org/0000-0002-5289-4806

\section{REFERENCES}

1 Reilly CM, Bruner DW, Mitchell SA, et al. A literature synthesis of symptom prevalence and severity in persons receiving active cancer treatment. Support Care Cancer 2013;21:1525-50.

2 Basch E, Dueck AC, Rogak LJ, et al. Feasibility assessment of patient reporting of symptomatic adverse events in multicenter cancer clinical trials. JAMA Oncol 2017;3:1043-50.

3 Enright K, Grunfeld E, Yun L, et al. Population-Based assessment of emergency room visits and hospitalizations among women receiving adjuvant chemotherapy for early breast cancer. J Oncol Pract 2015;11:126-32.

4 Sanoff HK, Carpenter WR, Freburger J, et al. Comparison of adverse events during 5-fluorouracil versus 5-fluorouracil/oxaliplatin adjuvant chemotherapy for stage III colon cancer: a population-based analysis. Cancer 2012;118:4309-20.

5 Chen J, Ou L, Hollis SJ. A systematic review of the impact of routine collection of patient reported outcome measures on patients, providers and health organisations in an oncologic setting. BMC Health Serv Res 2013;13:211.

6 Breen S, Ritchie D, Schofield P, et al. The Patient Remote Intervention and Symptom Management System (PRISMS) - a Telehealth- mediated intervention enabling real-time monitoring of chemotherapy side-effects in patients with haematological malignancies: study protocol for a randomised controlled trial. Trials 2015;16:472.

7 Howell D, Molloy S, Wilkinson K, et al. Patient-Reported outcomes in routine cancer clinical practice: a scoping review of use, impact on health outcomes, and implementation factors. Ann Oncol 2015;26:1846-58.

8 Moradian S, Voelker N, Brown C, et al. Effectiveness of Internetbased interventions in managing chemotherapy-related symptoms in patients with cancer: a systematic literature review. Support Care Cancer 2018;26:361-74.

9 Warrington L, Absolom K, Conner M, et al. Electronic systems for patients to report and manage side effects of cancer treatment: systematic review. J Med Internet Res 2019;21:e10875.

10 Mooney K, Whisenant MS, Beck SL. Symptom care at home: a comprehensive and pragmatic pro system approach to improve cancer symptom care. Med Care 2019;57:S66-72.

11 Gilbert JE, Howell D, King S, et al. Quality improvement in cancer symptom assessment and control: the provincial palliative care integration project (PPCIP). J Pain Symptom Manage 2012;43:663-78.

12 Basch E, Deal AM, Kris MG, et al. Symptom monitoring with patientreported outcomes during routine cancer treatment: a randomized controlled trial. J Clin Oncol 2016;34:557-65.

13 Basch E, Deal AM, Dueck AC, et al. Overall survival results of a trial assessing patient-reported outcomes for symptom monitoring during routine cancer treatment. JAMA 2017;318:197-8.

14 Beck SL, Eaton LH, Echeverria C, et al. SymptomCare@Home: Developing an Integrated Symptom Monitoring and Management System for Outpatients Receiving Chemotherapy. Comput Inform Nurs 2017;35:520-9.

15 Nixon NA, Spackman E, Clement F, et al. Cost-Effectiveness of symptom monitoring with patient-reported outcomes during routine cancer treatment. J Cancer Policy 2018;15:32-6.

16 Johansen MA, Henriksen E, Horsch A, et al. Electronic symptom reporting between patient and provider for improved health care service quality: a systematic review of randomized controlled trials. Part 1: state of the art. J Med Internet Res 2012;14:e118.

17 Hibbard JH, Mahoney E, Sonet E. Does patient activation level affect the cancer patient journey? Patient Educ Couns 2017;100:1276-9.

18 Papadopoulou C, Kotronoulas G, Schneider A, et al. PatientReported self-efficacy, anxiety, and health-related quality of life during chemotherapy: results from a longitudinal study. Oncol Nurs Forum 2017;44:127-36. 
19 National Cancer Institute. Common terminology criteria for adverse events, 2009

20 Kearney N, McCann L, Norrie J, et al. Evaluation of a mobile phonebased, advanced symptom management system (ASyMS) in the management of chemotherapy-related toxicity. Support Care Cancer 2009;17:437-44.

21 Stacey D, Green E, Ballantyne B, et al. Implementation of symptom protocols for nurses providing Telephone-Based cancer symptom management: a comparative case study. Worldviews Evid Based Nurs 2016;13:420-31.

22 Krzyzanowska MK, Walker-Dilks C, Atzema C, et al. Approach to fever assessment in ambulatory cancer patients receiving chemotherapy: a clinical practice guideline. Curr Oncol 2016;23:280-5.

23 Arain M, Campbell MJ, Cooper CL, et al. What is a pilot or feasibility study? A review of current practice and editorial policy. BMC Med Res Methodol 2010;10:67

24 Tickle-Degnen L. Nuts and bolts of conducting feasibility studies. Am J Occup Ther 2013;67:171-6.

25 Mooney KH, Beck SL, Friedman RH, et al. Automated monitoring of symptoms during ambulatory chemotherapy and oncology providers use of the information: a randomized controlled clinical trial. Support Care Cancer 2014;22:2343-50.

26 Portenoy RK, Thaler HT, Kornblith AB, et al. The Memorial symptom assessment scale: an instrument for the evaluation of symptom prevalence, characteristics and distress. Eur J Cancer 1994;30A:1326-36.

27 Henry JD, Crawford JR. The short-form version of the depression anxiety stress scales (DASS-21): construct validity and normative data in a large non-clinical sample. $\mathrm{Br} J$ Clin Psychol 2005;44:227-39.

28 Heitzmann CA, Merluzzi TV, Jean-Pierre P, et al. Assessing selfefficacy for coping with cancer: development and psychometric analysis of the brief version of the cancer behavior inventory $(\mathrm{CBI}-\mathrm{B})$. Psychooncology 2011;20:302-12.

29 Rabin R, de Charro F. EQ-5D: a measure of health status from the EuroQol group. Ann Med 2001;33:337-43.

30 Sauro J, Lewis JR. Chapter 8 - Standardized usability questionnaires. In: Quantifying the user experience. 2 edn. Boston: Morgan Kaufmann, 2016: 185-248.

31 Chan A-W, Tetzlaff JM, Gøtzsche PC, et al. Spirit 2013 explanation and elaboration: guidance for protocols of clinical trials. BMJ 2013;346:e7586.

32 Calvert M, Kyte D, Mercieca-Bebber R, et al. Guidelines for inclusion of patient-reported outcomes in clinical trial protocols: the SPIRITPRO extension. JAMA 2018;319:483-94.

33 Sandelowski M. Theoretical saturation. In: The SAGE encyclopedia of qualitative methods 1. CA: Thousand Oaks, 2008: 875-6.
34 Sharma MR, Stadler WM, Ratain MJ. Randomized phase II trials: a long-term investment with promising returns. J Natl Cancer Inst 2011;103:1093-100.

35 Cella DF, Tulsky DS, Gray G, et al. The functional assessment of cancer therapy scale: development and validation of the general measure. J Clin Oncol 1993;11:570-9.

36 Lenth RV. Some practical guidelines for effective sample size determination. Am Stat 2001;55:187-93.

$37 \mathrm{Sim}$ J, Lewis M. The size of a pilot study for a clinical trial should be calculated in relation to considerations of precision and efficiency. $J$ Clin Epidemiol 2012;65:301-8.

38 Billingham SAM, Whitehead AL, Julious SA. An audit of sample sizes for pilot and feasibility trials being undertaken in the United Kingdom registered in the United Kingdom clinical research network database. BMC Med Res Methodol 2013;13:104.

39 Stacey D, Carley M. The pan-Canadian Oncology Symptom Triage and Remote Support (COSTaRS)-Practice guides for symptom management in adults with cancer. Can Oncol Nurs J 2017;27:92-8.

40 Guest G, Bunce A, Johnson L. How many interviews are enough? an experiment with data saturation and variability. Field Methods 2006;18:59-82.

41 Latham JR. A framework for leading the transformation to performance excellence Part I: CEO perspectives on forces, facilitators, and strategic leadership systems. Qual Manag J 2013;20:12-33.

42 Greenbaum T. Moderating focus groups: a practical guide for group facilitation. Thousand Oaks, California, 2000. http://sk.sagepub.com/ books/moderating-focus-groups-a-practical-guide

43 Graneheim UH, Lundman B. Qualitative content analysis in nursing research: concepts, procedures and measures to achieve trustworthiness. Nurse Educ Today 2004;24:105-12.

44 Maguire R, Ream E, Richardson A, et al. Development of a novel remote patient monitoring system: the advanced symptom management system for radiotherapy to improve the symptom experience of patients with lung cancer receiving radiotherapy. Cancer Nurs 2015;38:E37-47.

45 Maguire R, Fox PA, McCann L, et al. The eSMART study protocol: a randomised controlled trial to evaluate electronic symptom management using the advanced symptom management system (ASyMS) remote technology for patients with cancer. BMJ Open 2017;7:e015016.

46 Moradian S, Krzyzanowska MK, Maguire R, et al. Usability evaluation of a mobile Phone-Based system for remote monitoring and management of chemotherapy-related side effects in cancer patients: mixed-methods study. JMIR Cancer 2018;4:e10932.

47 Stacey D, Macartney G, Carley M, et al. Development and evaluation of evidence-informed clinical nursing protocols for remote assessment, triage and support of cancer treatment-induced symptoms. Nurs Res Pract 2013;2013:11

$48 \mathrm{Sim}$ J. Should treatment effects be estimated in pilot and feasibility studies? Pilot Feasibility Stud 2019;5:107. 